\title{
Factores camión para diseño de pavimentos flexibles en Costa Rica: Análisis histórico en el período 2007-2017
}

\author{
Truck factors for flexible pavement design in Costa Rica: Historical analysis for the 2007-2017 period
}

\section{Jaime Allen, Ph.D.}

Unidad de Investigación en Infraestructura y Transporte Laboratorio Nacional de Materiales y Modelos Estructurales Universidad de Costa Rica

Costa Rica

jaime.allenmonge@ucr.ac.cr

\section{Eliécer Arias Barrantes, M.Sc.}

Unidad de Investigación en Infraestructura y Transporte Laboratorio Nacional de Materiales y Modelos Estructurales Universidad de Costa Rica

Costa Rica

eliecer.arias@ucr.ac.cr

\author{
Catalina Vargas Sobrado, M.Eng. \\ Unidad de Gestión Municipal \\ Laboratorio Nacional de Materiales y Modelos Estructurales \\ Universidad de Costa Rica \\ Costa Rica \\ anacatalina.vargas@ucr.ac.cr
}

\section{Allan Ureña Bermúdez}

Unidad de Investigación en Infraestructura y Transporte Laboratorio Nacional de Materiales y Modelos Estructurales Universidad de Costa Rica

Costa Rica

allan.urena@ucr.ac.cr

Fecha de recepción: 10 julio 2020 / Fecha de aprobación: 23 noviembre 2020

\section{RESUMEN}

El factor camión es uno de los componentes principales en la estimación de la demanda de cargas que tendrá el pavimento de una carretera. Por este motivo, disponer de factores camión confiables es de suma importancia durante la etapa de diseño. En el presente estudio se recopiló y analizó bases de datos sobre factores camión calculados entre los años 2007 y 2017, por el LanammeUCR. Se contabilizaron más de 15 millones de vehículos pesados, distribuidos en diferentes rutas del país. Dada la necesidad de disponer de factores camión actualizados que consideren la realidad nacional, tanto en la época sin control de pesaje como durante esta, se generan factores camión que están asociados a un nivel de confiabilidad específico y que consideran la proporción de vehículos que contribuyen en su estimación.

\section{ABSTRACT}

The truck factor is one of the main components in the estimation of the load demand that pavements will have. For this reason, having reliable truck factors is of utmost importance during the design stage. In the present study, the databases on truck factors calculated between 2007 and 2017 were compiled and analysed by LanammeUCR. More than 15 million heavy vehicles were analysed, distributed on different routes in the country. Given the need to have updated truck factors that take into account the national reality, both during the period without weight control and during the period with, truck factors are generated that are associated with a specific level of reliability and that consider the proportion of vehicles that contribute to their estimate.

Keywords: Reliability; pavement design; truck factor; weighing.

Palabras clave: Confiabilidad; diseño de pavimentos; factor camión; pesaje. 


\section{INTRODUCCIÓN}

Los factores camión que se utilizan actualmente en Costa Rica para el diseño de sobrecapas asfálticas y rehabilitaciones siguen las recomendaciones del oficio DVOP-5170-07, elaborado en el año 2007 por el Ing. Pedro Castro Fernández, entonces viceministro del Ministerio de Obras Públicas y Transportes (MOPT). El mismo se basó en la información recopilada a través de la encuesta de carga del año 2007, realizada por LanammeUCR (Laboratorio Nacional de Materiales y Modelos Estructurales [LanammeUCR], 2007; Ulloa Salazar, Badilla Vargas, Allen Monge y Sibaja Obando, 2008). Estos valores han sido ampliamente utilizados y corresponden al último pronunciamiento de la Administración respecto al tema de factores camión.

El Laboratorio Nacional de Materiales y Modelos Estructurales de la Universidad de Costa Rica (LanammeUCR) realiza investigaciones en el tema de cargas vehiculares desde el año 2006, con el propósito de generar insumos de diseño actualizados, y que representen las condiciones reales y actuales de carga a las cuales se ven sometidas las estructuras de pavimento. En consecuencia, el informe LM-PI-AT-121-2017 (LanammeUCR, 2018) evidenció que los factores camión recomendados en el oficio DVOP-5170-07, se encuentran desactualizados, e insta a la Administración a que adopte valores actualizados por el LanammeUCR.

Con esta motivación, entre los esfuerzos del LanammeUCR, se incluye determinar factores camión actualizados y establecer una actualización continua de los mismos que permita poner a disposición de la ciudadanía y la Administración factores camión adecuados a la flotilla vehicular del país y, por lo tanto, variables de diseño de mayor confiabilidad.

\section{Justificación}

La vida útil de una estructura de pavimento puede verse comprometida por diversos factores, entre ellos: (i) las condiciones del medio ambiente en que se encuentra, (ii) características de los materiales que la componen, (iii) cantidad de vehículos que la transitan y (iv) falencias durante su proceso constructivo. Sin embargo, uno de los factores más significativos corresponde al tránsito de vehículos pesados que superan su carga máxima admisible, pues si bien los vehículos pesados son aquellos que generan mayor daño a una estructura de pavimento, también se ha demostrado que cuando se genera un incremento de carga más allá de lo permitido, el daño crece de manera exponencial (Allen y Badilla, 2011).
Asimismo, una estimación poco precisa de las cargas de tránsito induce a sobrediseños, que si bien son resistentes, tienen un costo elevado; o por el contrario, a subdiseños, incapaces de soportar la demanda a la que serán sometidos, ocasionando así un deterioro prematuro. Por tanto, conocer y controlar las cargas vehiculares a través de la estimación de factores camión es sumamente importante para asegurar un uso eficiente de los recursos públicos empleados en el diseño de pavimentos, y por ende en la búsqueda de una maximización en su vida útil.

\section{Objetivo}

El objetivo principal de la presente investigación es determinar factores camión actualizados y acordes con la realidad de carga nacional, por medio del análisis del registro histórico de los factores camión generados por el LanammeUCR, en sus diferentes proyectos. Además, se propone incorporar la incidencia que tiene la cantidad de vehículos pesados en las diferentes estimaciones de factores camión, en los distintos estudios que se han realizado, considerando un nivel de confianza específico.

\section{Alcances}

Para la estimación de factores camión globales se utilizaron todas las bases de datos que ha recopilado el LanammeUCR, mediante encuestas de carga, un proyecto de graduación dirigido por personal del LanammeUCR, y datos brindados por el MOPT, asociados a las estaciones de pesaje que operan en las diferentes rutas de la red vial nacional y que han sido procesados en LanammeUCR. Así, se incluye en el análisis del estudio, las bases de datos asociadas a los años 2005-2007 de las encuestas de carga y las asociadas al período que comprende los años 2008-2017 de las estaciones de pesaje, además de otra fuente recopilada mediante trabajo final de graduación, específicamente para los vehículos tipo Bus-C2, del año 2013. Todos los pesajes se realizaron en rutas nacionales.

Este estudio pretende brindar una serie de factores camión actualizados y generales para el país, utilizando las bases de datos disponibles, buscando que estos estén asociados a un nivel de confianza específico de diseño, que permita contabilizar los factores camión actualizados acordes con la realidad nacional, sin dejar de lado el comportamiento histórico de los mismos. 


\section{Limitaciones}

Los factores camión estimados en este estudio son aplicables a diseños de pavimentos flexibles pues utilizan como referencia los factores equivalentes de carga ( $L E F$, por sus siglas en inglés) asociados a este tipo de pavimentos, estos representan la mayoría de los pavimentos de la red vial nacional, cerca de un 98 \% (LanammeUCR, 2019).

Los estudios considerados obedecen a distintas realidades nacionales asociadas a épocas donde existió un mayor y menor control de las cargas que transitan por las carreteras costarricenses. Sin embargo, ambas realidades aportan información valiosa al presente estudio, pues actualmente, son pocas las rutas que están sometidas a un régimen de control de cargas. Se recalca que es posible que algunos vehículos que circulan actualmente no sean contabilizados ya que utilizan vías alternas a los puestos de control de pesaje vigentes.

La cantidad de rutas consideradas es limitada, aproximadamente doce rutas. Sin embargo, los estudios se han enfocado en capturar el comportamiento de carga en diferentes rutas del país, todas ellas incluidas dentro de los corredores que compone el primer nivel jerárquico de la Red Vial Estratégica según el Plan Nacional de Transportes de Costa Rica (MOPT, 2011). Además, la cantidad total de vehículos considerados asciende a 15.386.304 de vehículos pesados. Los factores camión obtenidos en el presente estudio son representativos de las rutas más importantes del país y de aquellas rutas que cuentan con estaciones de pesaje y control para vehículos pesados.

\section{METODOLOGÍA}

Para obtener los valores recomendados de factores camión se siguieron las siguientes etapas metodológicas (ver Figura 1). Primero, se recopilaron las bases de datos disponibles y pertinentes para el estudio en cuestión, utilizando dos fuentes principales: información recopilada por el LanammeUCR, y registros históricos generados y procesados por el MOPT-LanammeUCR.

La encuesta de carga del LanammeUCR fue realizada durante los años 2005-2007, y publicada en el año 2007 con datos de pesajes manuales realizados en la Ruta 1, Ruta 2, Ruta 27, Ruta 32 y la Ruta 140 (LanammeUCR, 2008). Para estas rutas se consideraron los siguientes tipos de vehículos: liviano, $\mathrm{C} 2$, Bus-C2, C2+, C3 y T3-S2. Por otra parte, se tienen los datos recopilados a través de las estaciones de pesaje, para la Ruta 32 (2008-2017), Ruta 1 y Ruta 2 (entre los años 2009-2017), donde se analizaron los vehículos tipo C2, C3, C4, T3-S2 y T3-S3. Para la Ruta 32, Ruta 1 y Ruta 2 (entre los años 2008-2011) se analizaron los tipos de vehículos T2-S1 y T3-S1. Cada estudio y proyecto independiente contó con su propio alcance, por lo tanto, no se incluyen todos los vehículos posibles en todos los estudios, por este motivo se planteó utilizar todas las fuentes disponibles. Para el vehículo Bus-C2, se utilizó una base de datos de buses del proyecto de graduación de Espinoza (2013), pues los buses no se pesan en las estaciones de pesaje del MOPT. No se contabilizaron buses de tres ejes dado que representaban un porcentaje pequeño de los vehículos encuestados.
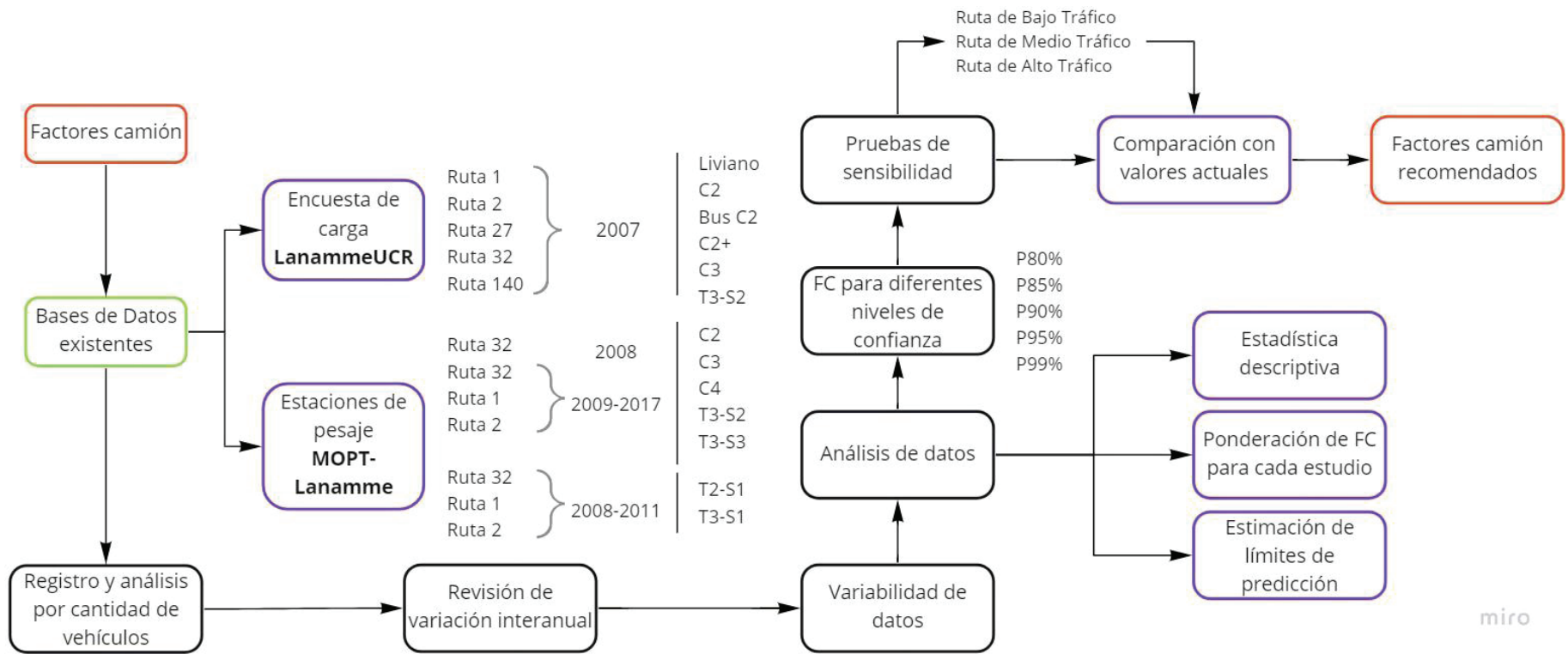

Figura 1. Esquema metodológico del estudio 
Como segundo paso, para las bases de datos mencionadas, después de hacer la recopilación, síntesis y análisis por cantidad de vehículos, se revisó la variación interanual de los factores camión, así como la variabilidad de los datos en general. Posteriormente se procedió a: (i) realizar una estadística descriptiva, (ii) generar una ponderación de los factores camión para cada uno de los estudios que conforman la información base del presente informe y (iii) generar una estimación de los límites de predicción de los factores camión.

Para las rutas de bajo tráfico ( $<5000$ veh/día), medio tráfico (5000 - 15000 veh/día) y alto tráfico (15 000 - 40000 veh/día), según la clasificación de tránsito elaborada por LanammeUCR (2008), se hicieron pruebas de sensibilidad de los datos y se compararon los valores obtenidos con los valores de los factores camión que se manejan actualmente por la Administración. Finalmente, se presentan los factores camión recomendados para el entorno nacional.

\section{RESULTADOS Y ANÁLISIS}

Se analizó un total de 15.386.304 vehículos que se clasifican en las siguientes ocho categorías: pick-up, C2, Bus-C2, C2+, C3, C4, T3-S2 y T3-S3 (ver Figura 2), para cuatro rutas nacionales, las cuales se componen de una ruta nacional de tránsito bajo (Paso Canoas, Ruta 2), dos rutas de tráfico medio
(Ruta 32 sentido Limón-San José y San José-Limón, y Ruta 2 sentido Ochomogo-San José y San José-Ochomogo), y una ruta de tránsito alto (Cañas, Ruta 1). Los vehículos T2-S1 y T3-S1 no se incluyeron dado que no se cuenta con suficiente información para considerar su variabilidad, su población es mínima en proporción al resto de los vehículos.

Dado que el objetivo del presente informe es brindar un factor camión asociado a un valor de confianza específico, se da una mayor ponderación a los factores que han sido calculados mediante estaciones de pesaje pues disponen de una mayor cantidad de datos (registro de vehículos). En contraparte, la encuesta de carga del 2007 registró una gran cantidad de rutas, pero una cantidad de vehículos limitada debido a que el pesaje se realizó de forma manual, vehículo por vehículo. Esto ofreció una ventana de observación interesante de factor camión, dado que en esta época no existía el control de pesaje por parte de la Administración.

A partir del análisis de la información disponible, se identifica que la estimación de factores camión para el tipo de vehículo C2 son mayoritariamente aportados por pesajes realizados en las rutas San José-Cartago y Cartago-San José; en tanto la mayor cantidad de registros de los vehículos T3-S2 y T3-S3 (correspondiente a vehículos articulados de 5 y 6 ejes), fueron recopilados principalmente en la Ruta 32 (Limón-San José, y San José-Limón).

\begin{tabular}{|c|c|}
\hline Tipo de vehículo & Pickup \\
\hline C2: Camión con eje dual trasero & \\
\hline Bus-C2: Bus con eje dual trasero & \\
\hline C2+: camión con eje simple trasero & \\
\hline C3: camión con eje tándem trasero & \\
\hline T3-S2: camión con eje tándem trasero & \\
\hline T3-S3: camión con eje trídem trasero
\end{tabular}

Figura 2. Categorías vehiculares utilizadas

Fuente: Ulloa et al., 2008 
La recopilación de datos desde el año 2008, permite analizar el comportamiento de los factores camión en el tiempo, tal y como se detalla en las Figura 3 y Figura 4, para las rutas nacionales Limón-San José y San José-Cartago, respectivamente. Las figuras muestran que, pese al establecimiento de estaciones de control de pesaje en el año 2008, aún no se define una tendencia a la estabilización clara o evidente para los factores camión en los distintos tipos de vehículos, a pesar de que otras rutas muestran menos variación interanual.
Asimismo, es importante considerar que entre los años 2008 y 2011 los pesajes no se llevaban a cabo durante las 24 horas del día, por lo que podría haber algún flujo de vehículos pesados no considerado en las estimaciones. Lo anterior dificulta las proyecciones de los factores camión, e induce a la consideración de un valor de confianza estadístico del resultado final asociado a la desviación estándar de la totalidad de las observaciones.

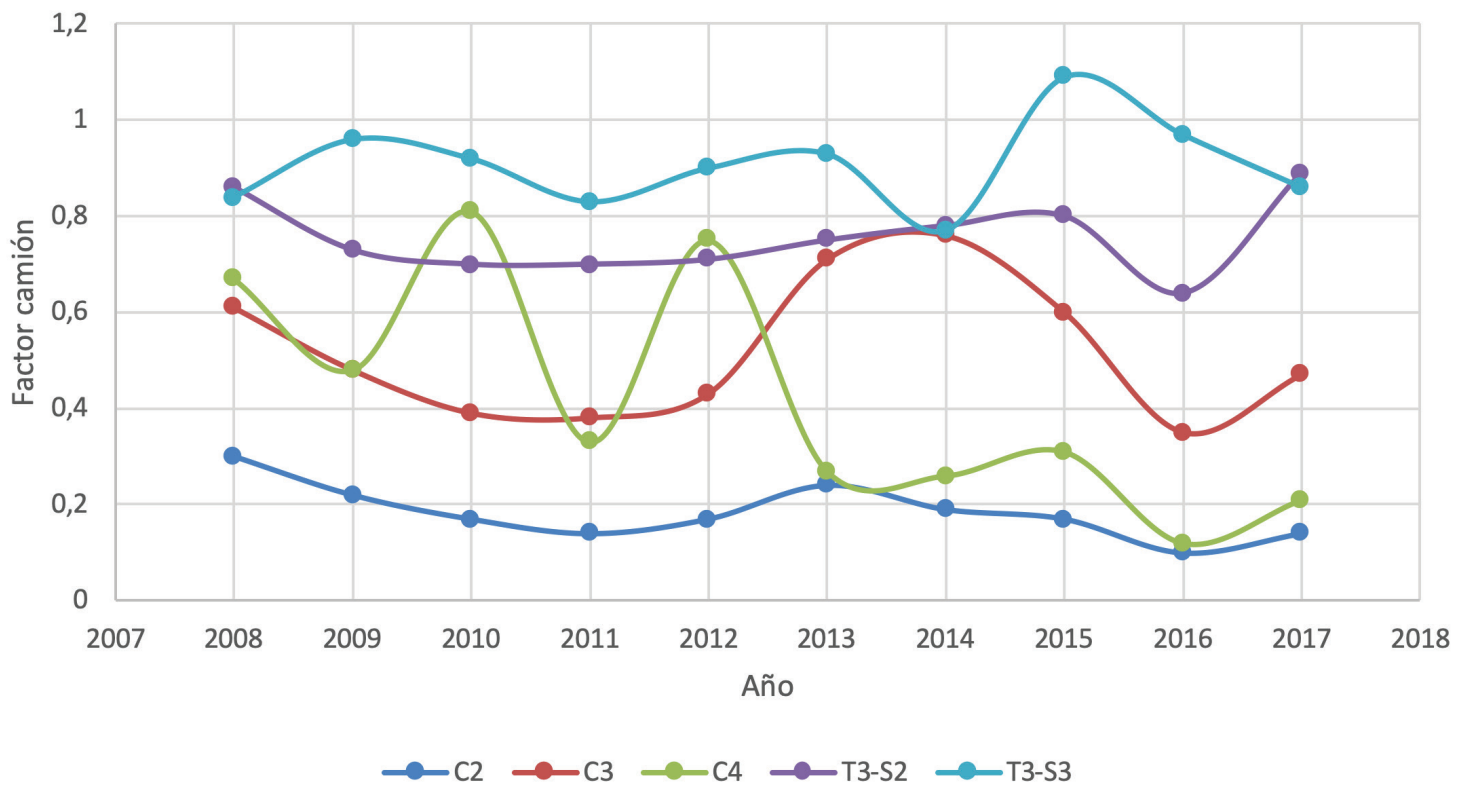

Figura 3. Factores camión promedio históricos en Ruta Nacional N32 sentido Limón-San José

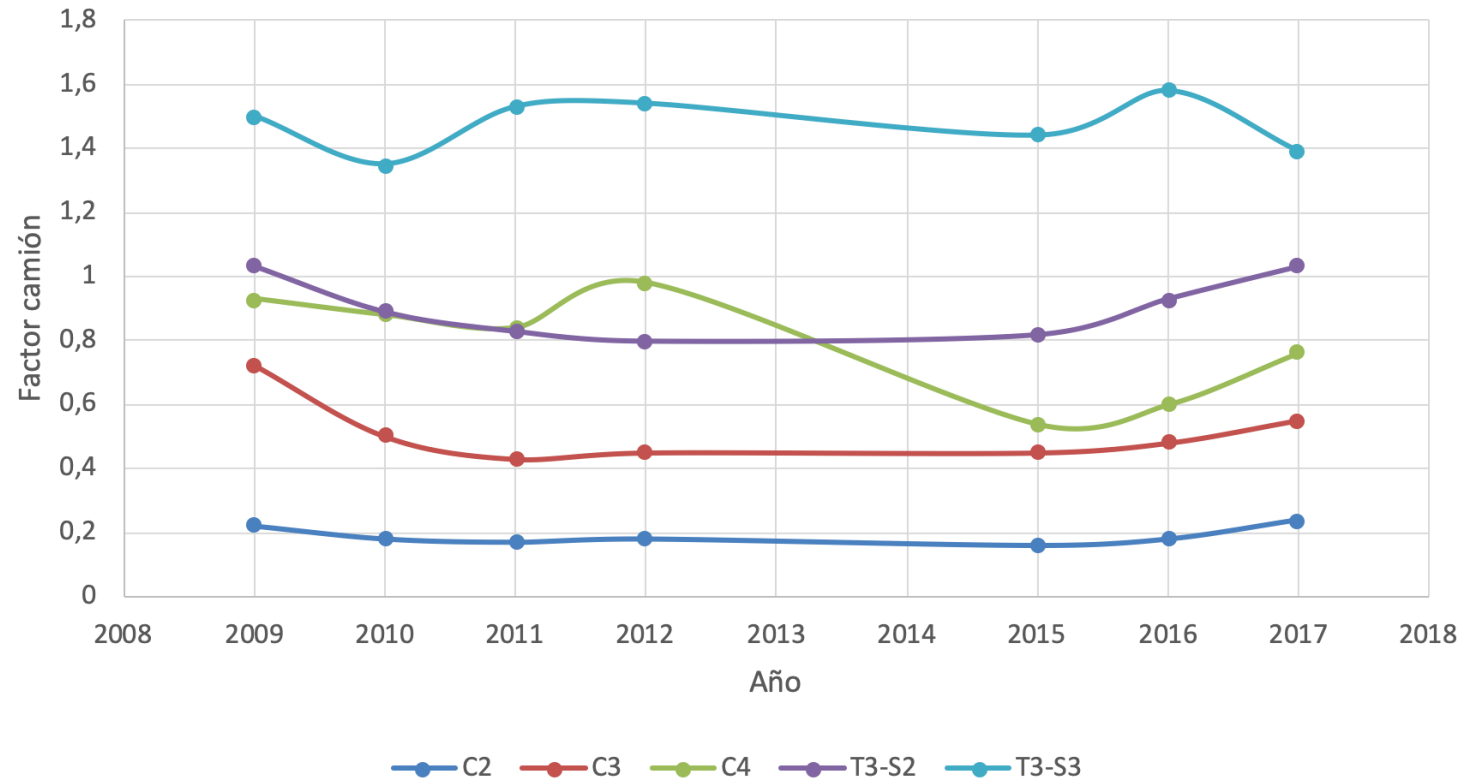

Figura 4. Factores camión promedio históricos en Ruta Nacional N 2 sentido San José-Cartago (Ochomogo) 
Esta variación anual de los factores camión requirió un análisis detallado del espectro de datos disponibles, la Cuadro 1 representa una estadística descriptiva de los datos. Los valores de los diferentes tipos de vehículos tienen rangos bastante amplios, es decir, tienen una gran separación entre su valor máximo y su valor mínimo, esto asociado también a una alta distancia promedio entre los valores y el valor medio (desviación estándar).

Esta variación se aprecia también mediante el coeficiente de variación que expresa la desviación estándar como porcentaje de la media aritmética, ya que expone el grado de variabilidad independientemente de la escala de la variable, a diferencia de la desviación estándar. Por ejemplo, según la Cuadro 1, para los vehículos tipo $\mathrm{C} 2$ se identifica un coeficiente de variación de $77,8 \%$, pese a que la media es 0,27 ; de igual manera sucede con los vehículos tipo C3, que presentan una media de 0,9 aunque reflejan un coeficiente de variación de $89,8 \%$. Para este análisis de datos la media aritmética no es representativa, ya que los factores camión obtenidos de los registros históricos presentan una alta variabilidad.

Por tanto, si en un futuro los factores camión estimados comenzaran a seguir una tendencia clara y definida, se podría considerar la selección de registros anuales y así minimizar la variabilidad presente en todos los registros históricos disponibles. Sin embargo, para la estimación de factores camión bajo el panorama actual, con alta variabilidad, se aplica el componente probabilístico con intervalos y límites de predicción (Ryan, 2007) a la matriz general de datos, asociada a niveles de confianza específicos.
Como se mencionó, el propósito del estudio es obtener una serie de factores camión ponderados por tipo de vehículo a partir de la cantidad de vehículos encuestados. Así, se utiliza la desviación estándar de los valores globales por tipo de vehículo para calcular factores camión que cumplan un nivel de confianza específico, según el siguiente modelo:

$$
F C_{V j P i}=F C_{V j}+\sigma_{j} Z_{i}
$$

donde

$\mathrm{FC}_{V j P i}=$ Factor camión para el vehículo j, dado un nivel de confianza i.

$\mathrm{FC}_{V j}=$ Factor camión ponderado para el vehículo j.

$\sigma_{j}=$ Desviación normal estándar para el vehículo j.

$\mathrm{Z}_{i}=$ valor de $\mathrm{z}$ asociado a un nivel de confianza i, para la distribución normal estándar.

Los factores camión obtenidos a partir de la Ecuación (1) para diferentes niveles de confianza se resumen en la Cuadro 2, cuya comparación con los últimos valores máximos y mínimos definidos por la Administración se presentan gráficamente en la Figura 5 y 6 . Esto demuestra que incluso la estimación de un factor camión con nivel de confianza de $85 \%$ para el caso de los vehículos tipo $\mathrm{C} 2+$, Bus-C2 y C3, supera el rango oficial establecido actualmente. Así, se ratifica la necesidad de implementar factores camión actualizados, que se ajusten a la realidad de la flota vehicular del país.

Cuadro 1. Descripción estadística de los factores camión estimados (2007-2017)

\begin{tabular}{|c|c|c|c|c|c|c|c|c|c|}
\hline $\begin{array}{c}\text { Tipo de } \\
\text { Vehiculo }\end{array}$ & Media & Mediana & Moda & $\begin{array}{c}\text { Desviación } \\
\text { estándar }\end{array}$ & Varianza & $\begin{array}{c}\text { Coeficiente de } \\
\text { variación }\end{array}$ & Rango & Mínimo \\
\hline C2 & 0,27 & 0,20 & 0,20 & 0,21 & 0,04 & $77,8 \%$ & 1,06 & 0,10 \\
\hline C2+ & 0,05 & 0,02 & 0,02 & 0,08 & 0,01 & $20,0 \%$ & 0,22 & 0,01 & 0,23 \\
\hline C3 & 0,88 & 0,54 & 0,43 & 0,89 & 0,79 & $89,8 \%$ & 3,44 & 0,33 & 3,77 \\
\hline C4 & 0,75 & 0,76 & 0,88 & 0,36 & 0,13 & $17,3 \%$ & 1,49 & 0,12 & 1,61 \\
\hline Bus C2 & 2,36 & 2,06 & - & 0,90 & 0,81 & $34,3 \%$ & 2,67 & 1,02 & 3,69 \\
\hline Pickup & 0,01 & 0,01 & 0,01 & 0,00 & 0,00 & $0,0 \%$ & 0,01 & 0,01 & 0,02 \\
\hline T2-S1 & 0,89 & 0,71 & - & 0,51 & 0,26 & $29,2 \%$ & 1,43 & 0,44 & 1,87 \\
\hline T3-S1 & 1,13 & 1,12 & - & 0,28 & 0,08 & $7,1 \%$ & 0,82 & 0,75 & 1,57 \\
\hline T3-S2 & 1,47 & 1,07 & 1,03 & 0,91 & 0,83 & $56,5 \%$ & 3,58 & 0,64 & 4,22 \\
\hline T3-S3 & 1,50 & 1,54 & 0,86 & 0,45 & 0,21 & $30,0 \%$ & 1,54 & 0,77 & 2,31 \\
\hline
\end{tabular}


Cuadro 2. Resumen de factores camión estimados (2007-2017) y valores utilizados por el MOPT actualmente

\begin{tabular}{|c|c|c|c|c|c|c|c|c|}
\hline \multicolumn{6}{|c|}{ Factor Camión Propuesto para diferentes } & \multicolumn{3}{|c|}{ Factor Camión MOPT } \\
\hline Vehículo & $80 \%$ & $85 \%$ & $90 \%$ & $95 \%$ & $99 \%$ & $\begin{array}{l}\text { Mínimo } \\
\text { MOPT }\end{array}$ & $\begin{array}{c}\text { Máximo } \\
\text { MOPT }\end{array}$ & Promedio \\
\hline Pickup & 0,013 & 0,013 & 0,014 & 0,014 & 0,015 & 0,010 & 0,020 & 0,010 \\
\hline $\mathrm{C} 2$ & 0,360 & 0,400 & 0,451 & 0,527 & 0,670 & 0,260 & 0,630 & 0,470 \\
\hline Bus C2 & 2,559 & 2,734 & 2,954 & 3,281 & 3,893 & 1,250 & 2,290 & 1,710 \\
\hline $\mathrm{C} 2+$ & 0,093 & 0,107 & 0,126 & 0,154 & 0,205 & 0,010 & 0,070 & 0,026 \\
\hline C3 & 1,250 & 1,414 & 1,621 & 1,928 & 2,504 & 0,990 & 1,280 & 1,100 \\
\hline C4 & 1,123 & 1,193 & 1,282 & 1,413 & 1,659 & & & \\
\hline T3-S2 & 1,920 & 2,098 & 2,321 & 2,652 & 3,273 & 1,510 & 2,380 & 1,710 \\
\hline T3-S3 & 2,142 & 2,230 & 2,341 & 2,506 & 2,815 & & & \\
\hline
\end{tabular}

Por tanto, de acuerdo con la revisión y análisis de los factores camión estimados para diferentes rutas del país y niveles tránsito vehicular, se recomienda como mínimo los valores de factores camión calculados con un $85 \%$ de confiablidad. Este nivel de confianza es comúnmente utilizado en el ámbito de la estadística ingenieril.

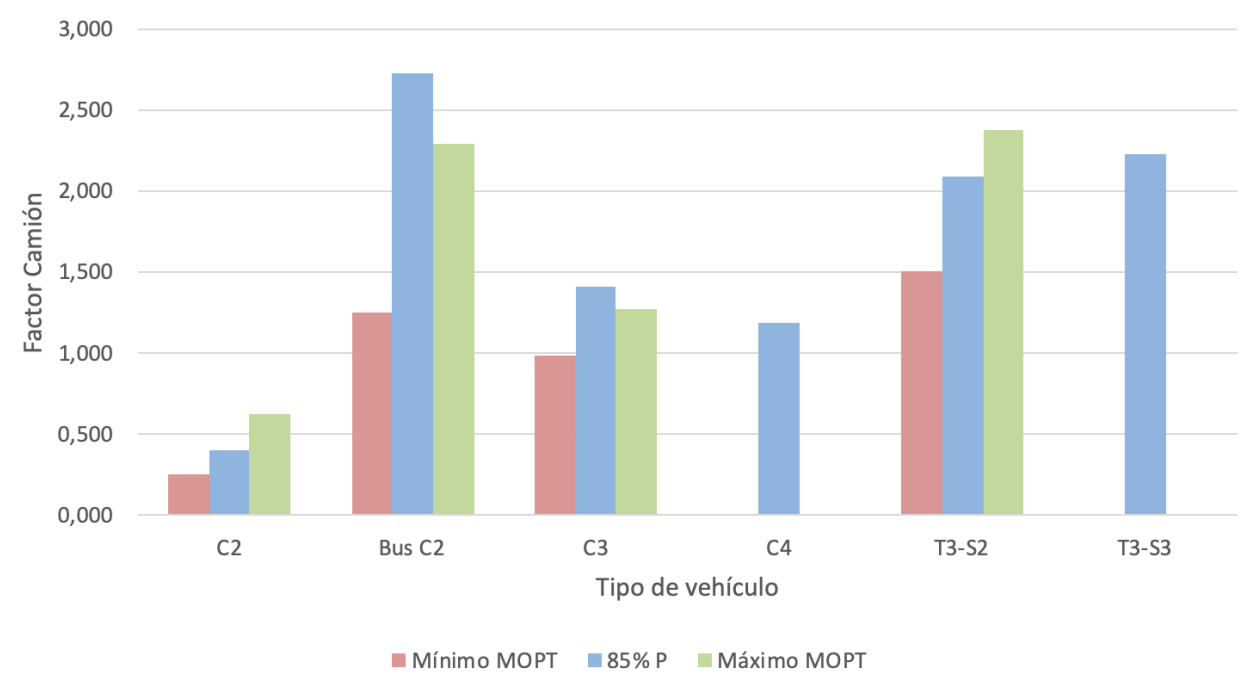

Figura 5. Factor camión para el 85\% de nivel de confianza y rangos del MOPT para los vehículos C2, Bus C2, C3, C4, T3-S2 y T3-S3

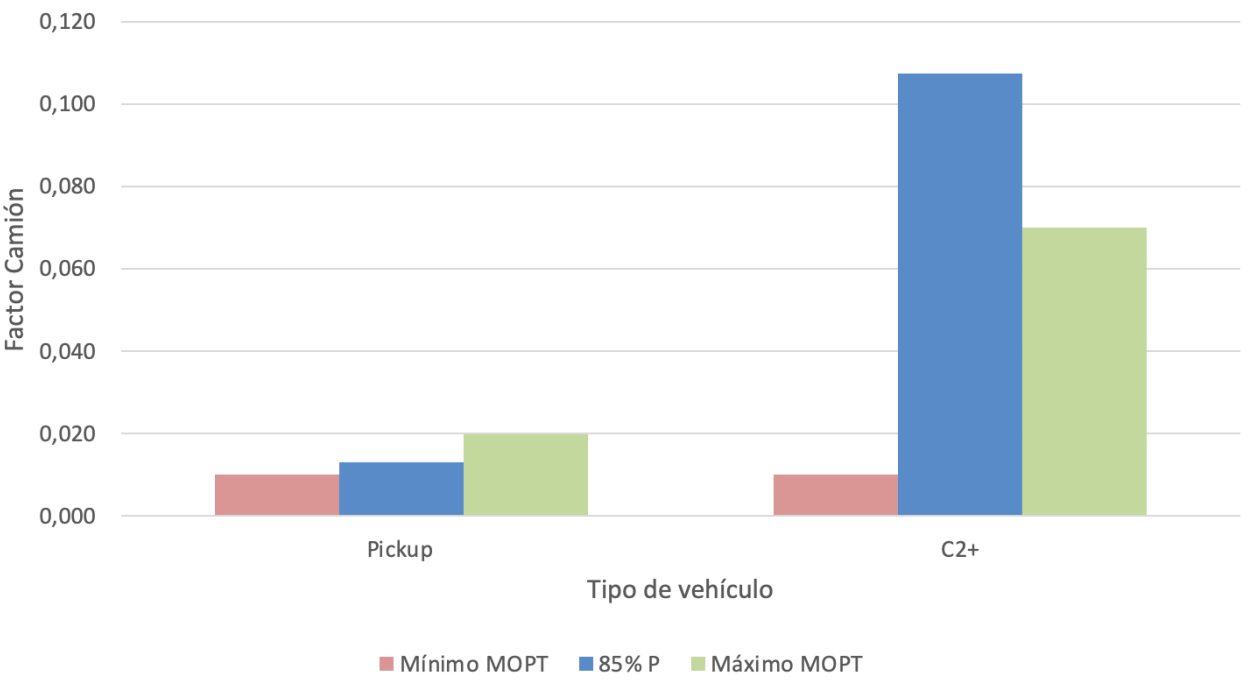

Figura 6. Factor camión para el 85\% de nivel de confianza y rangos del MOPT para los vehículos tipo C2+ y pickup 


\section{CONCLUSIONES Y RECOMENDACIONES}

Como valor agregado del análisis se obtiene la ponderación del factor camión por cantidad y tipo de vehículo, para analizar de forma integral los valores existentes y asociarlos a diferentes niveles de confianza. En los análisis realizados no se identifica una evidente estabilización del factor camión en Costa Rica, razón por la cual surge la necesidad de considerar la incidencia que posee la cantidad de vehículos de cada fuente de información, en su estimación.

Los valores acá expuestos, se calcularon a partir de una base de datos de más de 15 millones de vehículos, lo que brinda una cantidad de datos considerable para hacer estimaciones de variabilidad y nivel de confianza. Los valores de factor camión presentados se brindan como una alternativa a los valores que actualmente utiliza la Administración, dado que se obtuvieron con una mayor cantidad de datos, presentan mayor confiabilidad según los valores medidos hasta el año 2017 y capturan la variabilidad intrínseca del factor camión. Dentro de las ventajas que tiene la actualización de los factores camión y los valores finalmente recomendados se tienen las siguientes conclusiones:

- Los valores calculados representan las rutas con mayor cantidad de registros y tráfico del país en la actualidad.

- Los factores camión recomendados capturan información en escenarios que hoy se mantienen, sin control y con el mismo; por lo tanto, se puede inferir que son valores que van acorde con la realidad nacional.
- El análisis del presente estudio utiliza información de estaciones de pesaje, encuestas de carga y una tesis de grado, bases de datos disponibles al año 2017. Esto incorpora información adicional de 10 años en la estimación de factores camión, sobre aquellos correspondientes al último pronunciamiento oficial de la Administración en el año 2007.

- Los factores camión aquí presentados muestran una condición importante a considerar para la Administración, pues tal y como se demostró, incluso para un $85 \%$ de confianza, los factores camión actualizados de los vehículos tipo $\mathrm{C} 2+$, Bus-C2 y C3 superan los límites superiores establecidos por el Ministerio de Obras Públicas y Transportes, en su última actualización.

- Se recomienda actualizar la normativa en cuanto a los rangos de factores camión para diseño de pavimentos flexibles en Costa Rica, utilizando los obtenidos en el presente estudio.

Adicionalmente, se plantean recomendaciones para futuras investigaciones:

- Analizar estadísticamente el efecto de la implementación de las estaciones de pesaje en el factor camión, además de si existen cambios cuando varían las políticas de medición.

- Determinar si hay un efecto regional específico en los factores camión para los distintos vehículos de diseño, mediante el análisis de los datos en estaciones de pesaje individuales.

- Analizar el efecto temporal, horario y por período del año, en los factores camión para los distintos vehículos de diseño, mediante el análisis de los datos en estaciones de pesaje individuales.

\section{REFERENCIAS}

Allen, J. y Badilla, G. (2011). Determinación de la carga de diseño para pavimentos flexibles en Costa Rica. En XVI Congreso IberoLatinoamericano del Asfalto, Rio de Janeiro, Brasil, 2011. Recuperado de: https://www.lanamme.ucr.ac.cr/repositorio/ handle/50625112500/516

Espinoza González, J. C. (2013). Determinación de factores camión y espectros de carga representativos de autobuses urbanos e interurbanos para diseño de pavimientos flexibles en carreteras de Costa Rica (Tesis de licenciatura). Universidad de Costa Rica. San José, Costa Rica.

Laboratorio Nacional de Materiales y Modelos Estructurales (2007). Encuesta de Carga (PI-01-PIIVI-2007). Recuperado de https:// www.lanamme.ucr.ac.cr/repositorio/handle/50625112500/1473

Laboratorio Nacional de Materiales y Modelos Estructurales (2008). Propuesta de rangos para la clasificación de la Red Vial Nacional. Recuperado de https://www.lanamme.ucr.ac.cr/repositorio/handle/50625112500/1685

Laboratorio Nacional de Materiales y Modelos Estructurales (2018). Evaluación de parámetros y metodología utilizada en los Diseños de Pavimentos realizados bajo el marco de la Licitación Pública 2014LN-000018-0CV00 (LM-PI-AT-121-2017). Recuperado de https://www.lanamme.ucr.ac.cr/repositorio/handle/50625112500/1562 
Laboratorio Nacional de Materiales y Modelos Estructurales (2019). Informe de evaluación de la red vial nacional pavimentada de Costa Rica años 2018-2019 (LM-PI-UGERVN-004-19). Recuperado de https://www.lanamme.ucr.ac.cr/repositorio/ handle/50625112500/1750

Ministerio de Obras Públicas y Transporte (2007). DVOP-5170-07: Lineamientos diseño de pavimentos. San José, Costa Rica: Pedro Castro.

Ministerio de Obras Públicas y Transporte (2011). Plan Nacional de Transporte de Costa Rica 2011-2035. Recuperado de https:// www.mopt.go.cr/wps/wcm/connect/a86ca326-d0c2-403f-9166-259c356f4783/Memoria.pdf?MOD=AJPERES

Ryan, T. P. (2007). Modern Engineering Statistics. New Jersey, United States: John Wiley \& Sons.

Ulloa Salazar Á, Badilla Vargas G., Allen Monge J. y Sibaja Obando D. (2008). Determinación de factores camión en pavimentos de Costa Rica. Infraestructura Vial, 10(19), 28-37. Recuperado de https://revistas.ucr.ac.cr/index.php/vial/article/view/2048 